\title{
The Utility of Physicochemical Properties for the Prediction of Toxicological Outcomes: A Takeda
}

perspective.

Tomoya Yukawa and Russell Naven

Drug Safety Research and Evaluation, Pharmaceutical Research Division, Takeda Pharmaceutical Company Limited, 35 Landsdowne Street, Cambridge, MA, 02139, USA.

Drug Safety Research and Evaluation, Pharmaceutical Research Division, Takeda Pharmaceuticals International Company Limited, 9625 Towne Centre Drive, San Diego, CA 92121, USA.

Supporting information

$\checkmark$ Supplemental Table 1

$\checkmark$ Supplemental Table 2

$\checkmark$ Supplemental Table 3

$\checkmark$ Supplemental Table 4 
$\checkmark$ Experimental methods

Supplemental Table 1. Toxicity odds associated with the Takeda internal dataset. a) $1 \mu \mathrm{M}$ free concentration threshold of all data set, b) $1 \mu \mathrm{M}$ free concentration threshold of kinase inhibitors excluded data set.

\begin{tabular}{|c|c|c|c|c|c|}
\hline \multicolumn{3}{|c|}{ a) $C_{\max }$ (Free) analysis } & \multicolumn{3}{|c|}{ b) $C_{\max }$ (Free) analysis* } \\
\hline $\begin{array}{l}\text { Toxicity Odds } \\
\text { at } 1 \mu \mathrm{M}\end{array}$ & TPSA $>=75$ & TPSA $<75$ & $\begin{array}{l}\text { Toxicity Odds } \\
\text { at } 1 \mu \mathrm{M}^{*}\end{array}$ & TPSA $>=75$ & TPSA $<75$ \\
\hline $\operatorname{clog} \mathrm{P}<3$ & $0.49(55)$ & $0.18(20)$ & $c \log P<3$ & $0.23(38)$ & $0.18(20)$ \\
\hline $\operatorname{clog} \mathrm{P}>=3$ & $2.35(57)$ & $5.00(36)$ & $\operatorname{cog} P>=3$ & $2.06(49)$ & $4.50(33)$ \\
\hline
\end{tabular}

Supplemental Table 2. The compound number of each ionization state with physicochemical properties: ClogP vs TPSA.

\begin{tabular}{|c|c|c|c|c|c|c|c|c|c|c|c|c|c|}
\hline & & \multicolumn{12}{|c|}{ TPSA } \\
\hline & & \multicolumn{6}{|c|}{$>=75$} & \multicolumn{6}{|c|}{$<75$} \\
\hline & & \multicolumn{3}{|c|}{ clean } & \multicolumn{3}{|c|}{ tox } & \multicolumn{3}{|c|}{ clean } & \multicolumn{3}{|c|}{ tox } \\
\hline & & Acid & Base & Neutral & Acid & Base & Neutral & Acid & Base & Neutral & Acid & Base & Neutral \\
\hline \multirow{2}{*}{ ClogP } & $<3$ & 5 & 16 & 13 & 4 & 4 & 2 & 1 & 3 & 12 & 0 & 6 & 4 \\
\hline & $>=3$ & 12 & 5 & 22 & 9 & 25 & 4 & 4 & 3 & 9 & 1 & 15 & 8 \\
\hline
\end{tabular}

Supplemental Table 3. The compound number of each ionization state with physicochemical properties: ClogP vs 3D-PSA. 


\begin{tabular}{|c|c|c|c|c|c|c|c|c|c|c|c|c|c|}
\hline & & \multicolumn{12}{|c|}{ PSA } \\
\hline & & \multicolumn{6}{|c|}{$>=75$} & \multicolumn{6}{|c|}{$<75$} \\
\hline & & \multicolumn{3}{|c|}{ clean } & \multicolumn{3}{|c|}{ tox } & \multicolumn{3}{|c|}{ clean } & \multicolumn{3}{|c|}{ tox } \\
\hline & & Acid & Base & Neutral & Acid & Base & Neutral & Acid & Base & Neutral & Acid & Base & Neutral \\
\hline \multirow{2}{*}{$\mathrm{Clog} P$} & $<3$ & 6 & 18 & 18 & 4 & 6 & 1 & 0 & 0 & 7 & 0 & 4 & 5 \\
\hline & $>=3$ & 15 & 6 & 23 & 9 & 28 & 6 & 1 & 2 & 8 & 1 & 12 & 6 \\
\hline
\end{tabular}

Supplemental Table 4. The compound number of each ionization state with physicochemical properties: measured LogD vs TPSA.

\begin{tabular}{|c|c|c|c|c|c|c|c|c|c|c|c|c|c|}
\hline & & \multicolumn{12}{|c|}{ TPSA } \\
\hline & & \multicolumn{6}{|c|}{$>=75$} & \multicolumn{6}{|c|}{$<75$} \\
\hline & & \multicolumn{3}{|c|}{ clean } & \multicolumn{3}{|c|}{ tox } & \multicolumn{3}{|c|}{ clean } & \multicolumn{3}{|c|}{ tox } \\
\hline & & Acid & Base & Neutral & Acid & Base & Neutral & Acid & Base & Neutral & Acid & Base & Neutral \\
\hline \multirow{2}{*}{$\log D$} & $<2.5$ & 5 & 16 & 12 & 4 & 18 & 2 & 1 & 4 & 10 & 0 & 12 & 2 \\
\hline & $>=2.5$ & 11 & 4 & 20 & 9 & 11 & 4 & 4 & 2 & 9 & 1 & 8 & 10 \\
\hline
\end{tabular}

\section{Experimental methods}

The ClogP, and TPSA were calculated using Daylight version 4.95 (Daylight Chemical

Information Systems, Inc., Laguna Niguel, CA, USA). 
3D-PSA value (QikProp v 5.0, Schrödinger) was calculated by generating stable 3D conformations.

The pKa was calculated with ChemAxon 5.9. Acid/base classification was performed as described previously. The threshold was set at $\mathrm{pKa}=7$.

Log D7.4, which is the partition coefficient of the compounds between 1-octanol and aqueous buffer at $\mathrm{pH} 7.4$, was measured using a chromatographic procedure. ${ }^{25}$ 